九州大学学術情報リポジトリ

Kyushu University Institutional Repository

\title{
SILENT-NOISY DUEL WITH UNCERTAIN EXISTENCE OF
} THE SHOT

Teraoka, Yoshinobu

Department of Applied Mathematics, Himeji Institute fo Technology

https://doi.org/10.5109/13147

出版情報：統計数理研究. 19 (3/4)，pp.43-52，1981-03. Research Association of Statistical Sciences

バージョン :

権利関係 : 


\title{
SILENT-NOISY DUEL WITH UNCERTAIN EXISTENCE OF THE SHOT
}

\author{
By \\ Yoshinobu TERAOKA* \\ (Received October 20, 1980)
}

\begin{abstract}
The purpose of this paper is to solve a silent-noisy duel with arbitrary accuracy functions in which both duelists have an imperfect knowledge about the existence of the shot fitted to their gun. As a special case, the solution with only one shot and arbitrary accuracy function for each duelist will be shown.
\end{abstract}

\section{Introduction.}

In this paper we extend the silent-noisy duel in the work of Dresher [1963] and Karlin [1957] to include the uncertainty that both duelists have an imperfect knowledge about the existence of the shot fitted to their gun when the accuracy functions are arbitrary. The model is evidently formulated as a two-person zero-sum game defined on the unit square.

The silent-noisy duel has the following structure. Each player (i. e., duelist) can fire at any time in $[0,1]$ aming his opponent. For $i=1,2$, associated with Player $i$ is a continuous and strictly increasing function $A_{i}$, from $[0,1]$ onto $[0,1]$, called his accuracy function. If Player $i$ fires at time $t$, the probability that he hits his opponent, given that both players are still alive at $t$, is $A_{i}(t)$. Player I is the silent duelist and II is the noisy duelist, i. e., Player I knows whether Player II has fired or not, but not conversely. Let the payoff be +1 to the surviving duelist and 0 to each duelist if both survive or neither survive. Each selects a time to fire. This game with only one shot for each duelist under $A_{1}(t)=A_{2}(t)=t$ is described in the work of Dresher and Karlin.

Let us modify the above model by adding an assumption about the existence of the shot fitted to their gun. Suppose that $\theta_{i}$ is the number of shots fitted to the gun possessed by Player $i$ and that $\left(\theta_{1}, \theta_{2}\right)$ is a random variable with bivariate Bernoulli distribution given by Table 1 . The inner part of this double-entry table shows the joint distribution $\theta_{1}$ and $\theta_{2}$. Let $r$ be the correlation coefficent of $\theta_{1}$ and $\theta_{2}$ then $r=d / \sqrt{p_{1} q_{1} p_{2} q_{2}}$.

* Department of Applied Mathematics, Himeji Institute of Technology, 2167 Shosha, Himej, 671-22, Japan 
Table 1. Bivariate Bernoulli Distribution

\begin{tabular}{c|cc|c}
\multicolumn{3}{c}{$0<p_{i}=1-q_{i}<1,0 \leqq d \leqq \sqrt{p_{1} q_{1} p_{2} q_{2}}, 0 \leqq|d|<\min \left(p_{1} q_{2}, p_{2} q_{1}\right)$} \\
\hline$\theta_{1}$ & 1 & 0 & $\begin{array}{c}\text { Marginal } \\
\text { Distribution }\end{array}$ \\
\hline 1 & $\theta_{2}$ & $p_{1} q_{2}-d$ & $p_{1}$ \\
\hline $\begin{array}{c}\text { Marginal } \\
\text { Distribution }\end{array}$ & $p_{1} p_{2}+d$ & $q_{1} q_{2}+d$ & \\
\hline
\end{tabular}

We also assume that the accuracy functions $A_{1}$ and $A_{2}$ possess continuous derivatives with respect to $t$, which are denoted by $A_{1}^{\prime}(t)$ and $A_{2}^{\prime}(t)$, and that their first derivatives are strictly positive in $[0,1)$, for the sake of the argument in the remaining sections.

The silent and noisy duels under the same assumptions mentioned above have been solved by Teraoka [1975, for example, and 1976].

There are two interesting papers related to our model. Smith [1967] solved the restricted duel in which one player has a single noisy bullet while the other has both a silent and a noisy bullet and must fire the silent bullet first. Styszynski [1974] solved the silent vs. noisy duel when the accuracy functions are arbitrary the silent player has arbitrary (but nonrandom) numbers of shots and the noisy player has one.

Section 2 presents the payoff kernel suggested from our model and the fundamental lemma given by Karlin [1959]. In section 3 we shall discuss our main results, the unique optimal pair and the game value for our model. Section 4 duels with a simple example when $A_{1}(t)=A_{1}(t)=t$ and $d=0$.

\section{The formation.}

We suppose that both players are informed of the assumptions in Section 1. Each player does not know whether or not he has a shot, and is not informed of the number of shots fitted to his opponent's gun. However, a player in this game is forced to behave as if he has a shot and his maximum threat to his opponent is to fire as if he has a shot. Each selects a time to fire conditionally on his having one and that he survives. Furthermore one of the players has the advantage of being able to hear his opponent's shot.

If $x$ and $y$ are the firing times of Player I and II, respectively, the expected payoff to Player I for each state $\left(\theta_{1}, \theta_{2}\right)$ and its probability are given by Table 2 .

Hence the expected payoff kernel $M(x, y)$ for player I is

$$
M(x, y)= \begin{cases}p_{1} A_{1}(x)-p_{2} A_{2}(y)+\left(p_{1} p_{2}+d\right) A_{1}(x) A_{2}(y) & x<y \\ p_{1} A_{1}(x)-p_{2} A_{2}(x) & x=y \\ \left\{p_{1}-\left(p_{1} p_{2}+d\right)\right\} A_{1}(x)-\left\{p_{2}+\left(p_{1} p_{2}+d\right)\right\} A_{2}(y)+\left(p_{1} p_{2}+d\right) & x>y .\end{cases}
$$

Here, we shall state Lemma 1 which is given by Karlin [1959]. This lemma deals with a property of the optimal strategies for certain classes of timing games. 
Table 2. Expected Payoff for $\left(\theta_{1}, \theta_{2}\right)$ and Its Probability

\begin{tabular}{c|c|c|c|c}
\hline \multirow{2}{*}{$\left(\theta_{1}, \theta_{2}\right)$} & Probability & \multicolumn{3}{|c}{ Expected Payoff } \\
\cline { 3 - 4 } & & $x<y$ & $x=y$ & $x>y$ \\
\hline$(1,1)$ & $p_{1} p_{2}+d$ & $A_{1}(x)-A_{2}(y)+A_{1}(x) A_{2}(y)$ & $A_{1}(x)-A_{2}(x)$ & $1-2 A_{2}(y)$ \\
$(1,0)$ & $p_{1} q_{2}-d$ & $A_{1}(x)$ & $A_{1}(x)$ & $A_{1}(x)$ \\
$(0,1)$ & $q_{1} p_{2}-d$ & $-A_{2}(y)$ & $-A_{2}(y)$ & $-A_{2}(y)$ \\
$(0,0)$ & $q_{1} q_{2}+d$ & 0 & 0 & 0 \\
\hline
\end{tabular}

LEMMA 1. Let $M(x, y)$ be kernels of the form

$$
M(x, y)= \begin{cases}K(x, y) & x<y \\ \Phi(x, y) & x=y \\ L(x, y) & x>y\end{cases}
$$

which satisfy the following conditions:

(a) The functions $K(x, y)$ and $L(x, y)$ are defined over the closed triangles $0 \leqq x \leqq y \leqq 1$ and $0 \leqq y \leqq x \leqq 1$, respectively. Furthermore, they possess coutinuous second partial derivatives defined in their respective closed triangles.

(b) $K(1,1)>\Phi(1)>L(1,1)$ and $K(0,0) \leqq \Phi(0) \leqq L(0,0)$

(c) $K_{x}(x, y)>0$ and $L_{x}(x, y)>0$ for $x<1$,

$$
K_{y}(x, y)<0 \text { and } L_{y}(x, y)<0 \text { for } y<1
$$

(in their respective domains of definition).

Then optimal strategies of zero-sum game $M(x, y)$ exists uniquely and take the following from: densities $f(x), g(y)$ over a common support $[a, 1]$ and a possible jump at $I$ for one of the two players.

In the remaining sections, we suppose that I and II use mixed strategies (distribution functions over the unit interval), $F(x)$ and $G(y)$, respectively, and we define notations on expectation of $M(x, y)$ defined on the unit square as follows:

$$
\begin{aligned}
& M(F, G)=\int_{0}^{1} \int_{0}^{1} M(x, y) d F(x) d G(y) \text { and } \\
& M(x, G)=\int_{0}^{1} M(x, y) d G(y) ; \quad M(F, y)=\int_{0}^{1} M(x, y) d F(x) .
\end{aligned}
$$

For convenience, we also define $t_{0}$ to be the unique root of equation $A_{1}(t) A_{2}(t)+A_{1}(t)$ $+A_{2}(t)-1=0$ in the interval $[0,1]$, and define $h_{i}(t)$ by

$$
h_{i}(t)=A_{j}^{\prime}(t) /\left\{A_{1}(t) A_{2}(t)+A_{1}(t)+A_{2}(t)-1\right\} \quad \text { for } \quad t \in\left(t_{0}, 1\right),\{i, j\}=\{1,2\} .
$$

\section{The Solution.}

The kernel (1) clearly satisfies conditions in Lemma 1. Hence, we suppose that $F(x)$ consists of a density part $f(x)>0$ over an interval $[a, 1)$ and a mass part $\alpha$ at $x=1$, and $G(y)$ consists of a density part $g(y)>0$ on the same interval and a mass 
part $\beta$ at $y=1$.

Computing $M(F, y)$ from (1) and setting $\frac{d}{d y} M(F, y)=0$ yields

$$
\frac{\left(p_{1} p_{2}+d\right)\left\{1+A_{1}(y)\right\} f(y)}{p_{2}+\left(p_{1} p_{2}+d\right)-\left(p_{1} p_{2}+d\right) \int_{a}^{y}\left\{1+A_{1}(t)\right\} f(t) d t}=\frac{\left\{1+A_{1}(y)\right\} A_{2}^{\prime}(y)}{A_{1}(y) A_{2}(y)+A_{1}(y)+A_{2}(y)-1}
$$

which has solution

$$
f(x)=k_{1} h_{1}(x) \exp \left[-\int_{a}^{x}\left\{1+A_{1}(t)\right\} h_{1}(t) d t\right] \quad \text { for } \quad x \in[a, 1)
$$

In a similar fashion we obtain

$$
g(y)=k_{2} h_{2}(y) \exp \left[-\int_{a}^{y}\left\{1+A_{2}(t)\right\} h_{2}(t) d t\right] \quad \text { for } \quad y \in[a, 1) .
$$

Here we shall state some lemmas which are useful to determine the optimal strategies and the game value for game (1).

LEMMA 3. Given any $z>t_{0}$

$$
\int_{l}^{2}\left[\left\{1+A_{i}(t)\right\} A_{j}^{\prime}(t) /\left\{A_{1}(t) A_{2}(t)+A_{1}(t)+A_{2}(t)-1\right\}\right] d t \uparrow \infty \quad \text { as } \quad l \downarrow t_{0}(i \neq j) .
$$

And then $\exp \left[-\int_{l}^{z}\left\{1+A_{i}(t)\right\} h_{i}(t) d t\right]$ is a non-increasing function with resfect to $z$ for any fixed $l \in\left(t_{0}, 1\right)$.

Proof. We shall prove the case where $i=1$ and $j=2$. Since $t \geqq t_{0}$,

$$
\frac{\left\{1+A_{1}(t)\right\} A_{2}^{\prime}(t)}{A_{1}(t) A_{2}(t)+A_{1}(t)+A_{2}(t)-1} \geqq \frac{\left\{1+A_{1}\left(t_{0}\right)\right\} A_{2}^{\prime}(t)}{A_{1}(t) A_{2}(t)+A_{1}(t)+A_{2}(t)-1} .
$$

Using l'Hopital's rule, we obtain

$$
\begin{aligned}
& \lim _{t \rightarrow t_{0}}\left[\frac{\left\{1+A_{1}\left(t_{0}\right)\right\} A_{2}^{\prime}(t)}{A_{1}(t) A_{2}(t)+A_{1}(t)+A_{2}(t)-1} / \frac{\left\{1+A_{1}\left(t_{0}\right)\right\} A_{2}^{\prime}(t)}{A_{1}\left(t_{0}\right) A_{2}(t)+A_{1}\left(t_{0}\right)+A_{2}(t)-1}\right] \\
& =\lim _{t \rightarrow t_{0}} \frac{A_{1}\left(t_{0}\right) A_{2}(t)+A_{1}\left(t_{0}\right)+A_{2}(t)-1}{A_{1}(t) A_{2}(t)+A_{1}(t)+A_{2}(t)-1}=\frac{\left\{1+A_{1}\left(t_{0}\right)\right\} A_{2}^{\prime}\left(t_{0}\right)}{\left\{1+A_{1}\left(t_{0}\right)\right\} A_{2}^{\prime}\left(t_{0}\right)+\left\{1+A_{2}\left(t_{0}\right)\right\} A_{1}^{\prime}\left(t_{0}\right)}>0
\end{aligned}
$$

Hence there exists $s \in\left(t_{0}, z\right)$ such that

$$
\begin{aligned}
\frac{\left\{1+A_{1}\right.}{\left.\left.A_{1}(t) A_{0}\right)\right\} A_{2}^{\prime}(t)+A_{1}(t)+A_{2}(t)-1} \geqq & \frac{1}{2} \cdot \frac{\left\{1+A_{1}\left(t_{0}\right)\right\} A_{2}^{\prime}\left(t_{0}\right)}{\left\{1+A_{1}\left(t_{0}\right)\right\} A_{2}^{\prime}\left(t_{0}\right)+\left\{1+A_{2}\left(t_{0}\right)\right\} A_{1}^{\prime}\left(t_{0}\right)} \\
& \cdot \frac{\left\{1+A_{1}\left(t_{0}\right)\right\} A_{2}^{\prime}(t)}{A_{1}\left(t_{0}\right) A_{2}(t)+A_{1}\left(t_{0}\right)+A_{2}(t)-1}
\end{aligned}
$$

for any $t \in\left(t_{0}, s\right)$. Then we get

$$
\begin{aligned}
\int_{t_{0}}^{z} \frac{\left\{1+A_{1}(t)\right\} A_{2}^{\prime}(t)}{A_{1}(t) A_{2}(t)+A_{1}(t)+A_{2}(t)-1} d t \geqq & \frac{1}{2} \cdot \frac{\left\{1+A_{1}\left(t_{0}\right)\right\} A_{2}^{\prime}\left(t_{0}\right)}{\left\{1+A_{1}\left(t_{0}\right)\right\} A_{2}^{\prime}\left(t_{0}\right)+\left\{1+A_{2}\left(t_{0}\right)\right\} A_{1}^{\prime}\left(t_{0}\right)}- \\
& \cdot \operatorname{In}\left[\frac{A_{1}\left(t_{0}\right) A_{2}(s)+A_{1}\left(t_{0}\right)+A_{2}(s)-1}{A_{1}\left(t_{0}\right) A_{2}\left(t_{0}\right)+A_{1}\left(t_{0}\right)+A_{2}\left(t_{0}\right)-1}\right]=\infty,
\end{aligned}
$$


by breaking up the range of integration. This completes the proof of Lemma 2, since the integral is monotonically increasing as $l \downarrow t_{0}$.

Lemma 3. Putting

$$
U_{i}(z)=\exp \left[-\int_{a}^{z}\left\{1+A_{i}(t)\right\} h_{i}(t) d t\right] \quad \text { for } \quad i=1,2,
$$

the following equations hold

$$
\begin{aligned}
& \int_{l}^{u}\left\{1+A_{1}(x)\right\} f(x) d x=k_{1}\left\{U_{1}(l)-U_{1}(u)\right\} ; \\
& \int_{l}^{u}\left\{1+A_{2}(y)\right\} g(y) d y=k_{2}\left\{U_{2}(l)-U_{2}(u)\right\} .
\end{aligned}
$$

where $t_{0}<l \leqq u \leqq 1$, and $f(x)$ and $g(y)$ are given as (3) and (4), respectively.

PROOF. This can be shown easily, since $f(x)=k_{1} h_{1}(x) U_{1}(x)$ and $g(y)=k_{2} h_{2}(y) U_{2}(y)$.

LEMMA 4. The integral constants $k_{1}$ of $f(x)$ and $k_{2}$ of $g(y)$ take the following form

$$
p_{1}=\frac{p_{2}}{p_{1} p_{2}+d}+1 ; \quad k_{2}=\left\{\frac{p_{1}}{p_{1} p_{2}+d}-1+2 \beta\right\} / U_{2}(1), \quad \text { for } a \in\left(t_{0}, 1\right)
$$

where $\beta$ is the mass part of $G(y)$ at $y=1$.

PROOF. Inserting (3) in (2) yields $k_{1}$ stated in Lemma 4 . In a similar fashion we obtain $k_{2}$ in $(7)$.

LEMMA 5. For any fixed $\alpha \in[0,1)$ and $\beta \in[0,1)$, each of the equations on $a \in[0,1]$

$$
F(1-0)=\int_{a}^{1} f(x) d x=1-\alpha ; \quad G(1-0)=\int_{a}^{1} g(y) d y=1-\beta
$$

has a unique root in the interval $\left(t_{0}, 1\right)$.

Proof. For $a \in\left(t_{0}, 1\right), F(1-0)$ and $G(1-0)$ are strictly decreasing with respect to $a$, and $F(1-0)=G(1-0)=0$ at $a=1-0$. Since Lemma 3 holds, we have

$$
F(1-0) \geqq \frac{1}{2} \int_{a}^{1}\left\{1+A_{1}(x)\right\} f(x) d x=\frac{k_{1}}{2}\left\{1-U_{1}(1)\right\} \quad \text { for } \quad a \in\left(t_{0}, 1\right) .
$$

So that Lemmas 2 and 4 give

$$
F(1-0) \geqq \frac{1}{2}\left(\frac{p_{2}}{p_{1} p_{2}+d}+1\right)\left\{1-U_{1}(1)\right\} \uparrow \frac{1}{2}\left(\frac{p_{2}}{p_{1} p_{2}+d}+1\right)>1 \quad \text { as } \quad a \downarrow t_{0} .
$$

A similar argument on $G(1-0)$ shows

$$
G(1-0) \geqq \frac{1}{2}\left(\frac{p_{1}}{p_{1} p_{2}+d}-1+2 \beta\right)\left\{\frac{1}{U_{2}(1)}-1\right\} \uparrow \infty \quad \text { as } \quad a \downarrow t_{0} .
$$

This completes the proof of Lemma 5 .

By Lemmas 4 and 5 , each of the equations

$$
\int_{a}^{1}\left(\frac{p_{2}}{p_{1} p_{2}+d}+1\right) h_{1}(x) \exp \left[-\int_{a}^{x}\left\{1+A_{1}(t)\right\} h_{1}(t) d t\right] d x=1
$$




$$
\begin{array}{r}
\int_{a}^{1}\left\{\left(\frac{p_{1}}{p_{1} p_{2}+d}-1\right) h_{2}(y) \exp \left[-\int_{a}^{y}\left\{1+A_{2}(t)\right\} h_{2}(t) d t\right] /\right. \\
\left.\exp \left[-\int_{a}^{1}\left\{1+A_{2}(t)\right\} h_{2}(t) d t\right]\right\} d y=1
\end{array}
$$

has a unique root in the interval $\left(t_{0}, 1\right)$. Furthermote, $F(1-0)=1-\alpha$ and $G(1-0)=1-\beta$ give

$$
\begin{aligned}
& \alpha=1-\left(\frac{p_{2}}{p_{1} p_{2}+d}+1\right) \int_{a}^{1} h_{1}(x) \exp \left[-\int_{a}^{x}\left\{1+A_{1}(t)\right\} h_{1}(t) d t\right] d x \geqq 0 \\
& \beta=\frac{1-\left(\frac{p_{1}}{p_{1} p_{2}+d}-1\right) \int_{a}^{1} h_{2}(y) \exp \left[+\int_{a}^{1}\left\{1+A_{2}(t)\right\} h_{2}(t) d t\right] d y}{1+2 \int_{a}^{1} h_{2}(y) \exp \left[+\int_{y}^{1}\left\{1+A_{2}(t)\right\} h_{2}(t) d t\right] d y} \geqq 0
\end{aligned}
$$

for any $a$ greater than or equal to the roots of (8) and (9).

Elementary caluculation on $M(F, a)$ and $M(a, G)$ using Lemmas 3 and 4 gives the value of the game $v^{*}$ as follows:

$$
v^{*}=\left\{\begin{array}{cc}
\left\{p_{1}-\left(p_{1} p_{2}+d\right)\right\}\left\{p_{2}+\left(p_{1} p_{2}+d\right\}\left\{1-U_{1}(1)\right\} /\left(p_{1} p_{2}+d\right)\right. \\
-\left\{p_{2}+\left(p_{1} p_{2}+d\right)\right\} A_{2}(a)-p_{1}+2\left(p_{1} p_{2}+d\right), & \text { if } \alpha=0 \\
\left\{p_{1}-\left(p_{1} p_{2}+d\right)\right\}\left\{p_{2}-\left(p_{1} p_{2}+d\right) A_{1}(a)\right\} & \left.\alpha 1-1 / U_{2}(1)\right\} /\left(p_{1} p_{2}+d\right) \\
+\left\{p_{1}-\left(p_{1} p_{2}+d\right)\right\} A_{1}(a)+p_{2}, & \text { if } \beta=0 .
\end{array}\right.
$$

The above considerations lead us to Theorem 1 since Lemma 1 holds.

THEOREM 1. Let $a_{1}$ and $a_{2}$ be the unique roots of equations

$$
\begin{aligned}
& \int_{a_{1}}^{1} h_{1}(t) \exp \left[-\int_{a_{1}}^{t}\left\{1+A_{1}(s)\right\} h_{1}(s) d s\right] d t=\frac{p_{1} p_{2}+d}{p_{2}+\left(p_{1} p_{2}+p\right)} ; \\
& \int_{a_{2}}^{1} h_{2}(t) \exp \left[+\int_{t}^{1}\left\{1+A_{2}(s)\right\} h_{2}(s) d s\right] d t=\frac{p_{1} p_{2}+d}{p_{1}-\left(p_{1} p_{2}+d\right)}
\end{aligned}
$$

in the interval $\left(t_{0}, 1\right)$, respectively, and let $a=\max \left(a_{1}, a_{2}\right)$.

Then the optimal strategies of game $M(x, y), F^{*}(x)$ for I and $G^{*}(y)$ for II, are given by the following :

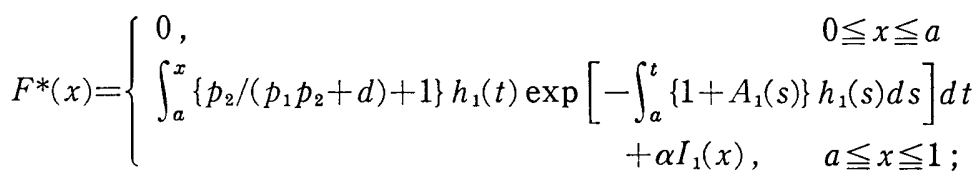

$$
\begin{aligned}
& G^{*}(y)=\left\{\begin{array}{c}
0, \\
\int_{a}^{y}\left\{p_{1} /\left(p_{1} p_{2}+d\right)-1+2 \beta\right\} h_{2}(t) \exp \left[+\int_{t}^{1}\left\{1+A_{2}(s)\right\} h_{2}(s) d s\right] d t \\
+\beta I_{1}(y), \quad a \leqq y \leqq 1
\end{array}\right.
\end{aligned}
$$

where $I_{1}(z)$ is the unit-step function at $z=1$, and mass parts $\alpha$ and $\beta$ are determined by 


$$
\begin{aligned}
& \alpha=1-\left(\frac{p_{2}}{p_{1} p_{2}+d}+1\right) \int_{a}^{1} h_{1}(t) \exp \left[-\int_{a}^{t}\left\{1+A_{1}(s)\right\} h_{1}(s) d s\right] d t \\
& \beta=\frac{1-\left(\frac{p_{1}}{p_{1} p_{2}+d}\right) \int_{a}^{1} h_{2}(t) \exp \left[+\int_{t}^{1}\left\{1+A_{2}(s)\right\} h_{2}(s) d s\right] d t}{1+2 \int_{a}^{1} h_{2}(t) \exp \left[+\int_{t}^{1}\left\{1+A_{2}(s)\right\} h_{2}(s) d s\right] d t} .
\end{aligned}
$$

Hence the next property holds:

$$
\alpha\left\{\begin{array}{l}
= \\
= \\
>
\end{array}\right\} 0 \text { and } \beta\left\{\begin{array}{l}
> \\
= \\
=
\end{array}\right\} 0 \text { if } a=\left\{\begin{array}{c}
a_{1}>a_{2} \\
a_{1}=a_{2} \\
a_{2}>a_{1}
\end{array}\right\} .
$$

The value of the game $v^{*}$ is given as

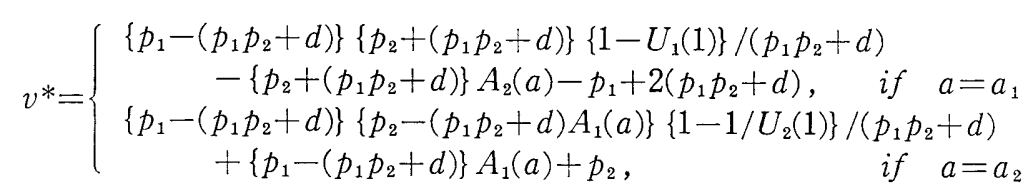

where $U_{i}(z)=\exp \left[-\int_{a}^{z}\left\{1+A_{i}(t)\right\} h_{i}(t) d t\right]$ for $z \in[a, 1], i=1,2$.

Letting $d=0, p_{1}=p_{2}$, and $p_{1} \rightarrow 1-0$, we have the case where each duelist has only one shot. Then

$$
\begin{gathered}
F^{*}(x)= \begin{cases}0 & 0 \leqq x<a \\
2 \int_{a}^{x} h_{1}(t) \exp \left[-\int_{a}^{t}\left\{1+A_{1}(s)\right\} h_{1}(s) d s\right] d t & a \leqq x \leqq 1 ;\end{cases} \\
G^{*}(y)= \begin{cases}0 & 0 \leqq y<a \\
\beta\left(2 \int_{a}^{y} h_{2}(t) \exp \left[+\int_{t}^{1}\left\{1+A_{2}(s)\right\} h_{2}(s) d s\right] d t+I_{1}(y)\right) & a \leqq y \leqq 1\end{cases}
\end{gathered}
$$

where $a$ is the unique root of equation

$$
\int_{a}^{1} h_{1}(t) \exp \left[-\int_{a}^{t}\left\{1+A_{1}(s)\right\} h_{1}(s) d s\right] d t=\frac{1}{2}
$$

in the interval $\left(t_{0}, 1\right)$ and mass part $\beta$ is given as

$$
\beta=1 /\left(1+2 \int_{a}^{1} h_{2}(t) \exp \left[+\int_{t}^{1}\left\{1+A_{1}(s)\right\} h_{1}(s) d s\right] d t\right)>0 .
$$

The value of the game $v^{*}$ is

$$
v^{*}=1-2 A_{2}(a) \text {. }
$$

This result is a special case of the result of Styszynski [1974]. 


\section{A Simple Example.}

As a simple example of Theorem 1, we shall examine the case where $d=0$ and $A_{1}(t)=A_{2}(t)=t$.

Since

$$
h_{i}(t)=1 /\left(t^{2}+2 t-1\right) \quad \text { and } \quad t_{0}=\sqrt{2}-1=0.414, \quad i=1,2
$$

we have for $z \in\left(t_{0}, 1\right)$

$$
U_{i}(z)=\sqrt{a^{2}+2 a-1} / \sqrt{z^{2}+2 z-1}, \quad i=1,2 .
$$

Hence $a_{1}$ and $a_{2}$ are the unique roots of equations

$$
\int_{a}^{1} \frac{\left(a^{2}+2 a-1\right)^{1 / 2}}{\left(t^{2}+2 t-1\right)^{3 / 2}} d t=\frac{p_{1}}{1+p_{1}} ; \quad \int_{a}^{1} \frac{\sqrt{2}}{\left(t^{2}+2 t-1\right)^{3 / 2}} d t=\frac{p_{2}}{1-p_{2}}
$$

which yield

$$
\sqrt{2} \sqrt{a^{2}+2 a-1}=a+\left(1-p_{1}\right) /\left(1+p_{1}\right) ; \quad \sqrt{2} \sqrt{a^{2}+2 a-1}=\left(1-p_{2}\right)(a+1)
$$

respectively.

It is found that $a_{1}$ and $a_{2}$ are the abscissas in $\{(a, b) \mid a \geqq 0, b \geqq 0\}$ of intersecting points of

hyperbola : $(a+1)^{2} / 2-b^{2} / 4=1$ and straight line: $b=a+\left(1-p_{1}\right) /\left(1+p_{1}\right)$;

hyperbola: $(a+1)^{2} / 2-b^{2} / 4=1$ and straight line: $b=\left(1-p_{2}\right)(a+1)$

respectively (see Fig. 1). Thus we get $a_{1}$ and $a_{2}$ are stsictly decreasing with respect to $p_{1}$ and $p_{2}$ respectively, and then

$$
t_{0}=\sqrt{2}-1<\sqrt{6}-2 \leqq a_{1}<1 ; \quad t_{0}=\sqrt{2}-1 \leqq a_{2}<1, \quad \text { for } 0<p_{1}, p_{2} \leqq 1 .
$$

Hence we get

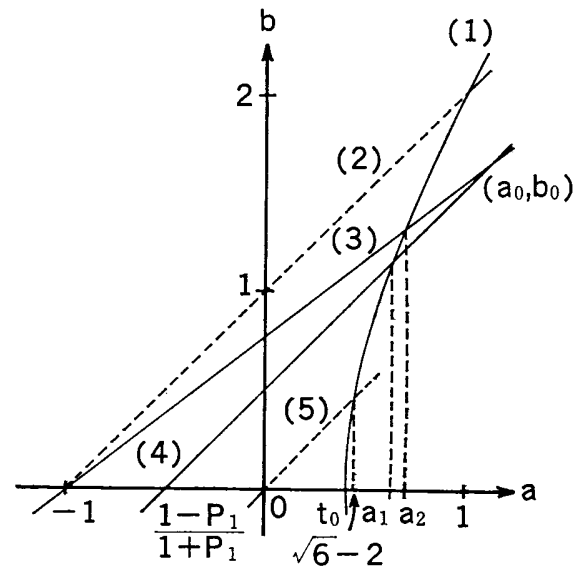

Fig. 1: (1): $\frac{(a+1)^{2}}{2}-\frac{b^{2}}{4}=1$

(2) : $b=a+1$

(3) : $b=\left(1-p_{2}\right)(a+1)$

(4) $: b=a+\left(1-p_{1}\right) /\left(1+p_{1}\right)$
(5): $b=a$ 


$$
a_{1}\left\{\begin{array}{l}
> \\
= \\
<
\end{array}\right\} a_{2} \quad \text { if } \quad \frac{\left(a_{0}+1\right)^{2}}{2}-\frac{b_{0}^{2}}{4}\left\{\begin{array}{l}
< \\
= \\
>
\end{array}\right\} 1
$$

where $\left(a_{0}, b_{0}\right)$ is the unique intersection of two lines:

which gives

$$
\left\{\begin{array}{l}
b=a+\left(1-p_{1}\right) /\left(1+p_{1}\right) \\
b=\left(1-p_{2}\right)(a+1)
\end{array}\right.
$$

$$
a_{0}=-1+\frac{2 p_{1}}{p_{2}\left(1+p_{1}\right)} \quad ; \quad b_{0}=\frac{2 p_{1}\left(1-p_{2}\right)}{p_{2}\left(1+p_{1}\right)} .
$$

After all we conclude that

$$
a=\left\{\begin{array}{c}
a_{1}>a_{2} \\
a_{1}=a_{2} \\
a_{2}>a_{1}
\end{array}\right\} \quad \text { if }\left(\frac{1+p_{1}}{p_{1}}\right)^{2}\left\{\begin{array}{c}
> \\
= \\
<
\end{array}\right\} \frac{2-\left(1-p_{2}\right)^{2}}{p_{2}^{2}},
$$

where

$$
a_{1}=\frac{2}{1+p_{1}}\left\{\sqrt{1+2 p_{1}+3 \overline{p_{1}^{2}}}-p_{1}\right\}-1 \quad ; \quad a_{2}=\left\{2 / \sqrt{1+2 p_{2}-p_{2}^{2}}\right\}-1
$$

The open unit square $\left\{\left(p_{1}, p_{2}\right) \mid 0<p_{1}<1,0<p_{2}<1\right\}$ is devided into two regions (in one of which $a=a_{1}$ and, in the other $a=a_{2}$ ) as shown in Fig. 2.

Hence the densities over common interval $[a, 1]$ of $F^{*}(x)$ and $G^{*}(y)$ are

$$
\begin{aligned}
& f^{*}(x)=\left(\frac{1}{p_{1}}+1\right) \frac{\left(a^{2}+2 a-1\right)^{1 / 2}}{\left(x^{2}+2 x-1\right)^{3 / 2}} ; \\
& g^{*}(y)=\left(\frac{1}{p_{2}}-1+2 \beta\right) \frac{\sqrt{2}}{\left(y^{2}+2 y-1\right)^{3 / 2}}
\end{aligned}
$$

respectively.

Using (16), (18), and (19), $\alpha, \beta$ and $v^{*}$ are the following:

(i) if $a=a_{1}$, then

$$
\alpha=0 \text {, }
$$

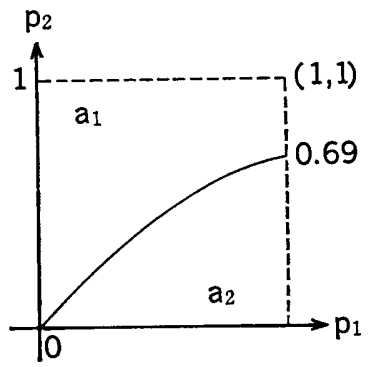

Fig. 2: The region for initial firing time where $A_{1}(t)=A_{2}(t)=t, d=0$. 


$$
\begin{aligned}
\beta & =\left[a+1-2 p_{1} /\left\{p_{2}\left(1+p_{1}\right)\right\}\right] /\left[a+2-\left(1-p_{1}\right) /\left(1+p_{1}\right)\right] \\
& =1-p_{1}\left(1+1 / p_{2}\right) /\left(1+2 p_{1}+3 p_{1}^{2}\right)^{1 / 2} \\
v^{*} & =(1 / 2)\left[1+p_{1}-p_{2}+p_{1} p_{2}-\left(1+p_{1}\right)\left(1+p_{2}\right) a\right] \\
& =1+2 p_{1}\left(1+p_{2}\right)-\left(1+p_{2}\right)\left(1+p_{1}+3 p_{1}^{2}\right)^{1 / 2}
\end{aligned}
$$

(ii) if $a=a_{2}$, then

$$
\begin{aligned}
\alpha & =1-(1 / 2) p_{2}\left\{\left(1 / p_{1}\right)+1\right\}(a+1)=1-p_{2}\left(1+p_{1}\right) /\left\{p_{1}\left(1+2 p_{2}-p_{2}^{2}\right)^{1 / 2}\right\}, \\
\beta & =0, \\
v^{*} & =1-2\left(1-p_{1} a\right) /(1+a)=1+2 p_{1}-2\left(1+p_{1}\right) /(1+a) \\
& =1+2 p_{1}-\left(1+p_{1}\right)\left(1+2 p_{2}-p_{2}^{2}\right)^{1 / 2} .
\end{aligned}
$$

\section{Acknowledgement}

The author is very grateful to Professor M. Sakaguchi of Osaka University for his guidance and encouragement. Furthermore he heartily thanks to the referees for their many helpful comments and suggestions.

\section{References}

[1] Dresher, M.: Games of Strategy: Theory and Applications. Prentiəe Hall, New York, 1963.

[2] Karlin, S.: Mathematical Methods and Theory in Games, Programming and Economics. Vol. II, Addison-Wesley, New York, 1959.

[3] Sмith, G.: A duel with silent-noisy gun versus noisy gun. Colloq. Math. 17 (1967), 131146.

[4] Styszynski, A.: An n-silent-vs.-noisy duel with arbitrary accuracy functions. Zastos. Math. 14 (1974), 205-225.

[5] TERAOKA, Y.: Silent duel with uncertain shot. Reports of H.I.T., No. 28. A, (1975), 1-8.

[6] TeraokA, Y.: Correction to silent duel with uncertain shot. Reports of H.I.T., No. 29. A, (1976), 1-4.

[7] TeraokA, Y.: Noisy duel with uncertain existence of the shot. Int. J. Game Theory, 5 (1976), 239-249.

[8] TERAOKA, Y: A two-person game of timing with random arrival time of the object. Math. Japonica, 24 (1979), 427-438. 